\title{
Centro de Operações de Emergência da Secretaria Estadual de Saúde do Paraná - Pandemia Covid-19
}

\author{
Emergency operations center of Health Depart- \\ ment of State of Paraná - Pandemic Covid-19
}

\begin{abstract}
Maria Goretti David Lopes ${ }^{1}$, Luciane Otaviano de Lima², Acácia Maria Lourenço Francisco $\mathrm{Nasr}^{3}$, Sabrina Requião Pinto ${ }^{4}$, Elaine Drehmer de Almeida Cruz ${ }^{5}$

1. ORCID: https://orcid.org/0000-0002-6782-4640. Especialista em Planejamento e Desenvolvimento de Recursos Humanos para a Saúde. Secretaria do Estado da Saúde do Paraná (Curitiba, Paraná, Brasil). E-mail: maria.lopes@sesa.pr.gov.br.

2. ORCID: https://orcid.org/0000-0002-0885-3687. Mestre em Ciências Farmacêuticas. Secretaria do Estado da Saúde do Paraná (Curitiba, Paraná, Brasil). E-mail: luciane.lima@sesa.pr.gov.br.

3. ORCID: https://orcid.org/0000-0002-7663-6094. Mestre em Tocoginecologia. Secretaria do Estado da Saúde do Paraná (Curitiba, Paraná, Brasil). E-mail: acacianasr@gmail.com.

4. ORCID: https://orcid.org/0000-0002-1590-3910. Mestre em Engenharia Civil. Secretaria do Estado da Saúde do Paraná (Curitiba, Paraná, Brasil). E-mail: sabrina.requiao@sesa.pr.gov.br.

5. ORCID: https://orcid.org/0000-0002-7686-6340. Doutora em Enfermagem. Universidade Federal do Paraná e Secretaria do Estado da Saúde do Paraná. (Curitiba, Paraná, Brasil). E-mail: elainedrehmercruz@gmail.com.
\end{abstract}

CONTATO: Elaine Drehmer de Almeida Cruz | Telefone: (41) 984168951 | E-mail: elainedrehmercruz@gmail.com | Rua Piquiri, nº 170, Rebouças, Curitiba - PR. CEP 80230-140

RESUMO Este artigo apresenta o planejamento e ações de enfrentamento à pandemia por Covid-19 pelo Centro de Operações de Emergência em Saúde Pública da Secretaria Estadual de Saúde do Paraná. Trata-se de relato de experiência relativo ao processo de desenvolvimento e produtos decorrentes de ações de enfrentamento da pandemia relativos ao período de fevereiro a junho de 2020. As ações desenvolvidas resultaram na aprovação de 51 documentos direcionados à organização do enfrentamento da pandemia e apreciados em mais de 70 reuniões presenciais. Os produtos estão apresentados em assuntos: Medidas Administrativas de Enfrentamento; Controle Sanitário; 
Populações Vulneráveis e Serviços Especializados de Saúde; Processo de Trabalho; Rastreamento Laboratorial, Tratamento e Promoção da Saúde; e Dados Epidemiológicos. As ações, experiência e aprendizagem alcançadas pelo Centro de Operações contribuíram para o enfrentamento da pandemia no Estado e contribuirão para enfrentamentos futuros.

DESCRITORES: Planos de Emergência. Gestão de Riscos. Infecções por Coronavírus. Pandemias.

\begin{abstract}
This paper presents the planning and actions to combat the Covid-19 pandemic of the Center for Emergency Operations in Public Health of the State Health Department of Paraná. This is an experience report related to the development process and products resulting from actions to combat the pandemic concerning the period from February to June 2020. The actions developed resulted in the approval of 51 documents targeted to the organization of facing the pandemic and appreciated in more than 70 presential meetings. The products are presented in subjects: Administrative Measures of Coping; Sanitary Control; Vulnerable Populations and Specialized Health Services; Work Process; Laboratorial Tracking, Treatment and Health Promotion; and Epidemiological Data. The actions, experience, and learning achieved by the Operations Center contributed to facing the pandemic in the State and will contribute to future confrontations.
\end{abstract}

DESCRIPTORS: Emergency Plans. Risk Management. Coronavirus Infections. Pandemics.

\title{
INTRODUÇÃO
}

E m 11 de março de 2020 a Organização Mundial da Saúde declarou estado de pandemia, pelo então denominado novo coronavírus, mediante sua disseminação em mais de 100 países ${ }^{1}$. Embora tenha sido o marco legal para que os Estados-Membros da Organização adotassem medidas legais em relação ao risco, de acordo com o Regulamento Sanitário Internacional², as administrações governamentais brasileiras haviam se mobilizado, antecipadamente, para o enfrentamento desta situação de emergência em saúde pública.

Detectado em 30 de dezembro de 2019, o surto por doença respiratória na China rapidamente se disseminou além das fronteiras, sendo o agente etiológico identificado como novo coronavírus, nominado SARS-CoV-2. A doença Covid-19 
tem rápida evolução para Síndrome Respiratória Aguda Grave, e potencialidade para alta mortalidade entre grupos especiais, embora ainda pouco conhecido o tratamento eficaz.

Com base em experiências anteriores, com surtos e epidemias gripais, o $\mathrm{Mi}$ nistério da Saúde ativou, em 22 de janeiro de 2020, o Centro de Operações de Emergência em Saúde Pública (COE-Covid-19), cujo objetivo foi nortear resposta do Sistema Único de Saúde (SUS) à possível emergência de saúde pública. Progressivamente, outras ações legais foram instituídas ou acionadas, como o Grupo Executivo Interministerial e o Grupo de Emergência em Saúde Pública, a fim de estabelecer ações de enfrentamento. A situação de Emergência Nacional foi declarada em 3 de fevereiro e, no decorrer do mesmo mês, a primeira morte pela doença foi anunciada no dia 17 e o primeiro caso de Covid-19 foi confirmado no dia $26^{3}$. Ainda em fevereiro, o Ministério da Saúde publicou o Plano de Contingência Nacional para Infecção Humana pelo novo Coronavírus, definindo o nível de resposta e a estrutura de comando correspondente em cada nível ${ }^{4}$. O reconhecimento da transmissão comunitária se deu no dia 23 de março de $2020^{5}$.

No Paraná, em 2 de fevereiro, considerando o Plano de Resposta às Emergências em Saúde estadual ${ }^{6}$, foi ativado o Centro de Operações de Emergência em Saúde Pública da Secretaria Estadual de Saúde do Paraná - COE. Este tem por objetivo definir as estratégias e procedimentos para o enfrentamento da pandemia, a fim de reduzir os potenciais impactos para a saúde pública, por meio de resposta coordenada, eficaz, eficiente e oportuna?

Neste contexto histórico, este relato de experiência tem por objetivo apresentar o planejamento e ações de enfrentamento à pandemia por Covid-19 na Secretaria do Estado da Saúde do Paraná.

\section{MÉTODO}

Trata-se de estudo descritivo documental de abordagem qualitativa ${ }^{8}$, do tipo 
relato de experiência, que descreve e contextualiza a atuação da Secretaria do Estado da Saúde do Paraná (SESA-PR) no planejamento e desenvolvimento de ações de enfrentamento à pandemia Covid-19. O relato refere-se ao período de fevereiro a junho de 2020, correspondente desde a ativação do $\operatorname{COE}^{7}$ em 2 de fevereiro até 30 de junho de 2020, data do Decreto Governamental $4942^{9}$, que dispõe sobre medidas restritivas regionalizadas para o enfrentamento da pandemia.

A reunião dos documentos se deu na página eletrônica da SESA-PR e registros das reuniões do COE, esses apresentados e contextualizados pelas autoridades e técnicos coparticipantes do planejamento e ações.

Esta publicação atende às normativas da Resolução do Conselho Nacional de Saúde $n^{\circ} 510$, de 7 de abril de 2016, e considerando tratar-se de documentos públicos, ora apresentados e discutidos, seu conteúdo não foi submetido à apreciação por Comitê de Ética em Pesquisa.

\section{RESULTADOS E DISCUSSÃO}

A ativação do COE, pelo Secretário de Estado da Saúde do Paraná, se deu por meio da Resolução SESA n 126/2020, com o objetivo de reduzir potenciais impactos da situação de emergência em saúde pública, por meio de resposta coordenada, eficaz, eficiente e oportuna7. O COE, inicialmente coordenado por representante do Centro de Informações Estratégicas de Vigilância em Saúde - CIEVS/ $\mathrm{PR}$, foi composto pelos seguintes serviços e entidades da SESA-PR': Coordenadorias de Vigilância Epidemiológica; de Organização de Rede de Cuidados da Saúde, de Vigilância Ambiental, de Promoção da Saúde, de Vigilância Sanitária, de Regulação do Acesso aos Serviços de Saúde, Centro de Informações Estratégicas em Vigilância em Saúde; Divisão de Vigilância de Doenças Transmissíveis; Gerências de Atenção Primária em Saúde, de Urgência e Emergência; Laboratório Central do Estado do Paraná; Núcleo de Comunicação Social; Vigilância de Saúde do Trabalhador; Comissão de Avaliação, Auditoria e Monitoramento; Ouvidoria Geral 
da Saúde; e Gabinete do Secretário de Estado da Saúde.

A primeira reunião aconteceu no dia 13 de fevereiro, com a presença, in loco, de sete pessoas. Considerando o teor da referida Resolução, que permite acionar outros setores e instituições para participação, em 19 de março de 2020 foi publicada a Resolução n ${ }^{\circ} 317 / 2020^{10}$, e o COE passou a ser coordenado pela Diretoria de Atenção e Vigilância em Saúde. Sempre que necessário, as reuniões contaram com a participação de representantes do Conselho de Secretarias Municipais de Saúde; Agência Nacional de Vigilância Sanitária; Autarquia dos Portos; Ministério Público; Entidades Profissionais e Sociedades Científicas; Representantes da Rede Privada de Assistência à Saúde; entre outras.

A periodicidade das reuniões foi determinada de acordo com a magnitude e complexidade dos eventos. De fevereiro até junho de 2020 foram realizadas 74 reuniões, com a participação presencial na sede da SESA-PR, e online por meio da disponibilização de link de acesso, totalizando neste período 1.181 participantes presenciais. As reuniões ocorreram, na maioria, no período vespertino de segundas às sextas-feiras, com pauta estabelecida diariamente e comunicada com antecipação. O modelo dos encontros se dá com apresentação inicial dos dados epidemiológicos do Paraná, do Brasil e do mundo.

Em relação ao Estado do Paraná, são apresentados os números de casos e de óbitos, novos e total, por regional de saúde, sexo e faixa etária. Os pontos de pauta seguintes são apresentados e a reunião é finalizada com informes. A cada assunto tratado é oportunizada a participação dos presentes e membros virtuais, bem como apresentação de perguntas e sugestão de encaminhamentos, além de proposição de assuntos para pauta das reuniões subsequentes.

O COE é importante alavanca, junto a outras instâncias da administração, para a busca de estratégias de enfrentamento da pandemia. Deste modo, além de ser momento oportuno para identificar ações pertinentes ao controle da pandemia, também consiste em fórum para avaliação das medidas a serem adotadas no âmbito estadual, assim como de documentos a serem publicizados, particular- 
mente, as notas orientativas e técnicas, relativas aos mais diferentes contextos.

Fruto desta demanda de enfrentamento da situação epidemiológica da Covid-19 na esfera estadual, procedimentos e estratégias foram traçados e operacionalizados. Em conjunto, no período de quatro meses foram apresentados e aprovados pelo COE 41 Normas Orientativas correlatas à pandemia, seis minutas de resolução, duas notas técnicas e uma nota conjunta. Os documentos, com as versões atualizadas, e a seguir apresentadas, podem ser acessados na página da SESA-PR, pelo endereço eletrônico http://www.saude.pr.gov.br/Pagina/Coronavirus-COVID-19.

Considerando a diversidades das ações, para fins de organização deste relato de experiência estão apresentadas nos tópicos: Medidas Administrativas de Enfrentamento, Controle Sanitário, Equipamentos de Proteção, Populações Vulneráveis e Serviços Especializados de Saúde, Processo de Trabalho, Rastreamento Laboratorial, Tratamento e Promoção da Saúde, e Dados Epidemiológicos.

\section{Medidas administrativas de enfrentamento}

A primeira medida administrativa e legal publicada foi a Resolução SESA $n^{\circ}$ 0126/20207, em 2 de fevereiro de 2020, com a ativação do COE para o enfrentamento do coronavírus, então denominado 2019-nCoV.

Em 20 de março de 2020 foi publicada a Resolução SESA n 338/202011 que implementa medidas de enfrentamento da emergência em saúde pública de importância nacional e internacional decorrente do Coronavírus - Covid-2019 e atende ao disposto Decreto $n^{\circ} 4230 / 2020$, do Governo Estadual ${ }^{12}$, o qual determinou a adoção das referidas medidas. Nesse documento destacam-se identificação e isolamento de doentes, a suspensão de eventos abertos ao público e a suspensão das férias de servidores estaduais. Em 25 de maio, nova resolução prorrogou os prazos estabelecidos na resolução anterior, enquanto subsistissem as medidas de enfrentamento da emergência em saúde pública decorrente da Covid-19. 
Em 24 de março de 2020 foi publicada a Resolução $n^{\circ} 340 / 2020^{13}$, que estabelece ações para contratação emergencial e institui recursos de custeio para oferta de leitos de Unidade de Terapia Intensiva e de Retaguarda Clínica para atendimento de usuários do SUS. Esta resolução foi fundamental diante da demanda esperada por internamento hospitalar em curto prazo. Ainda neste contexto, mediante a demanda prevista por leitos de UTI e maior necessidade de ventiladores mecânicos, a Nota Orientativa $n^{\circ} 35 / 2020^{14}$ foi publicada com o objetivo de abordar os regulamentos sanitários aplicáveis na fabricação e importação de ventiladores pulmonares no contexto da pandemia da Covid-19, considerando as excepcionalidades e esclarecimentos aplicados pela Agência Nacional de Vigilância Sanitária.

\section{Controle sanitário}

As medidas de prevenção e controle sanitário constituem um dos pilares fundamentais no enfrentamento da pandemia, e em decorrência disso ações foram desenvolvidas em diferentes contextos. Dentre essas foram elaboradas orientações regulatórias quanto à fabricação, importação e comercialização de produtos de interesse à saúde, como equipamentos de proteção individual e ventiladores pulmonares que tiveram aumento de demanda em decorrência da Covid-19; tratativas referentes a medicamentos controlados; notificações de possíveis eventos adversos e queixas técnicas; regulamentações específicas de controle sanitário, além das ações de fiscalização para mitigação de riscos identificados.

Neste contexto, desde o início da pandemia foram produzidas, diretamente, 20 Notas Orientativas que abordam, majoritariamente, os aspectos sanitários, além da transversalidade do tema em outras Notas que também trouxeram abordagens sobre esses aspectos.

Em relação, especificamente, às medidas abrangentes e gerais de controle sanitário que podem ser aplicadas aos mais variados contextos, citam-se a limpeza e desinfecção de ambientes, preparações antissépticas e sanitizantes, prevenção da propagação da Covid-19 em residências, orientações para confecção de más- 
caras para população, e desinfecção de locais públicos (Nota Orientativa n ${ }^{\circ}$ 01, 02, 16, 22 e 23, respectivamente) ${ }^{14}$. Demais medidas de controle sanitário descritas permeiam outros tópicos, visto que estão relacionadas com equipamentos de proteção individual, processos de trabalho e tratamento.

Ainda como forma de apoio ao enfrentamento da pandemia e tendo em vista a atribuição da Vigilância Sanitária no contínuo processo de licenciamento sanitário, a Resolução SESA no $544 / 2020^{15}$ foi publicada dispondo procedimentos para prorrogação do prazo de validade das Licenças Sanitárias no Estado do Paraná em decorrência da pandemia.

Considerando a necessidade de regulamentação das medidas complementares de controle sanitário a serem adotadas pelos locais autorizados a funcionar no Paraná, foi publicada a Resolução SESA $n^{0} 632^{16}$, com destaque para medidas específicas de distanciamento social, higienize de mãos, limpeza e desinfecção, comunicação e orientações gerais de prevenção, bem como as restrições aplicadas ao momento.

\section{Populações vulneráveis e serviços especializados de saúde}

Em acordo com as evidências disponíveis, a partir do exemplo de outros países e da realidade nacional, sabe-se que determinados grupos populacionais estão mais vulneráveis ao vírus SARS-CoV-2 e cuja doença se apresenta de forma mais grave. Cientes deste contexto e com vistas a antecipar-se ao risco, cinco Notas Orientativas foram publicadas e direcionadas a esses grupos em especial ${ }^{14}$.

A Nota Orientativa $n^{\circ} 04 / 2020^{13}$ trata do atendimento dos idosos frente à pandemia Covid-19, e reitera a importância atribuída a essa população, especialmente vulnerável frente ao envelhecimento. Assim como, cuidados direcionados no atendimento em oncologia diante da pandemia, publicados na Nota Orientativa n $18 / 2020$, considerando a população com imunidade deprimida pela doença de base, e a Nota Orientativa $n^{\circ}$ 11/2020 direcionada às medidas de prevenção frente ao tabagismo e uso de derivados do tabaco ${ }^{14}$. 
As Notas Orientativas $n^{\circ} 27$ e $n^{\circ}$ 32/2020 abordam, respectivamente, medidas de prevenção e controle para serviços de diálise e os cuidados às pessoas com Hipertensão Arterial Sistêmica e Diabetes Mellitus diante da pandemia ${ }^{14}$. Sabe-se que indivíduos portadores de comorbidades são mais vulneráveis à Covid-19 e, portanto, são merecedores de atenção especial. Os serviços de diálise necessitam de especial organização, tanto para a prevenção da transmissão do vírus, quanto para a manutenção dessa terapia em pacientes com diagnóstico suspeito ou confirmado da virose. Neste aspecto, também doentes crônicos em atendimento ambulatorial foram objeto da Nota Orientativa $n^{\circ} 33 / 2020^{14}$, direcionada para os Ambulatórios Organizados no modelo de atenção às condições crônicas.

\section{Processo de trabalho}

A pandemia impôs mudanças no processo de trabalho, em inúmeras situações, exigindo olhar particularizado de acordo com diferentes formas de trabaIhar, ambientes e profissionais com atividades distintas, ao se pretender orientar medidas de prevenção e controle assertivas.

Neste cenário desafiador, foram publicadas 17 Notas Orientativas ${ }^{14}$ e uma Resolução ${ }^{17}$, cujos objetivos foram normatizar princípios para o controle do ambiente e do processo de trabalho, com vistas a reduzir o risco de transmissão do vírus entre pessoas. Considerando a demanda por Equipamento de Proteção Individual diante da pandemia e a necessidade de esclarecimentos e orientações quanto à fabricação e uso dos mesmos, foi publicada a Nota Orientativa $n^{\circ}$ 03/2020 que aborda o uso de máscaras para proteção, a Nota Orientativa n 12/2020 que trata da fabricação desses equipamentos e a Nota Orientativa n 26/2020 que discorre sobre a importação, comercialização e doação de produtos para saúde destinados à Covid-19, dentre os quais os equipamentos de proteção ${ }^{14}$.

Foi publicada a Nota Orientativa $n^{0} 13 / 2020^{14}$ que direciona orientações aos empregadores e trabalhadores sobre a prevenção do coronavírus nos ambientes de trabalho, com exceção dos estabelecimentos de saúde.

Para serviços em geral de atendimento ao público, foram elaboradas as Notas 
Orientativas $n^{\circ}$ 06/2020 (mercados, supermercados, hipermercados, atacarejos e todos os outros estabelecimentos que comercializem alimentos), $n^{\circ}$ 07/2020 (serviços de alimentação), n 08/2020 (delivery de alimentos), n 28/2020 (refeitórios destinados à alimentação de funcionários e colaboradores), e n 34/2020 (shopping centers, centros comerciais e galerias) ${ }^{14}$. Neste mesmo sentido foi publicada a Resolução SESA n ${ }^{\circ} 856 / 2020^{17}$ que trata das normas para realização de atividades religiosas.

Para serviços de saúde e de interesse à saúde, foram publicadas as Notas Orientativas nº5/2020 (farmácias que dispensam medicamento do componente especializado e complementar), $n^{\circ}$ 09/2020 (cuidado materno infantil), $n^{\circ}$ 19/2020 (manejo de óbitos), n² 20/2020 (transporte sanitário), n²1/2020 (atendimento pré-hospitalar móvel de urgência e transporte interinstitucional), $\mathrm{n}^{\circ}$ 24/2020 (orientações para adequação da estrutura física em caráter temporário), n $25 / 2020$ (centrais de abastecimento farmacêutico das regionais de saúde da SESA-PR), n' 29/2020 (puericultura), $n^{\circ}$ 38/2020 (prevenção e controle da transmissão horizontal de infecção) e $n^{\circ} 39 / 2020$ (atendimento odontológico) ${ }^{14}$.

Considerando-se como ambiente de especial risco aos trabalhadores, a Nota Orientativa $n^{\circ} 31 / 2020^{14}$ trata de recomendações para a indústria de abate e processamento de carnes (frigoríficos) para o enfrentamento à pandemia da Covid-19. E a Resolução SESA n ${ }^{\circ} 855^{18}$, publicada e posteriormente revogada, tratava da adoção de amplas medidas, agregando o disposto nas notas anteriormente publicadas, com destaque para a vigilância e busca ativa de casos, com afastamento imediato e implantação de medidas de rastreabilidade de contatos; organização do trabalho e uso de EPI; oportunidade de trabalho remoto para grupo de risco e garantia de afastamento de trabalhadores, entre outras.

\section{Rastreamento laboratorial, tratamento e promoção da saúde}

O rastreamento laboratorial na rede do Sistema Único de Saúde no Paraná foi incentivado por meio da distribuição de testes rápidos, recebidos do Ministério da Saúde, e cujos critérios de uso foram divulgados por meio da Nota Técnica ${ }^{\circ}$ 
05/2020 ${ }^{19}, \mathrm{n}^{\circ}$ 07/202020 e complementação ${ }^{21}$.

Com vistas a ampliar a testagem na capital do Estado, uma ação conjunta entre a SESA-PR e o Complexo Hospital do Trabalhador viabilizou a disponibilidade de testagem por meio de drive thru (Nota Técnica Conjunta DAV/CHT SESA $01 / 2020^{22}$ ), especialmente direcionada aos trabalhadores de saúde e de segurança pública, e seus contatos familiares.

Considerando a comercialização e realização de testes rápidos nas farmácias, e de modo a normatizar essa prática, foi publicada a Resolução SESA n 781/202023.

Com vistas a ampliar a testagem da população, a Nota Orientativa $n^{\circ} 40^{14}$ trata do rastreamento laboratorial da Covid-19 e condutas de afastamento do trabaIho. E a Nota Orientativa n ${ }^{\circ} 41 / 2020^{14}$ apresenta medidas de prevenção, controle e testagem de idosos e trabalhadores de instituições de longa permanência para idosos.

Quanto ao tratamento de pacientes acometidos pela Covid-19, e considerando o isolamento social, a Resolução SESA no 482/202024 regulamentou, em caráter excepcional e temporário, a operacionalização de prescrição médica por meio eletrônico. Frente à nota informativa ministerial, a Secretaria publicou a Nota Orientativa $n^{\circ} 37 / 2020^{14}$ sobre manuseio medicamentoso precoce de pacientes com diagnóstico de Covid-19.

Regulamentos sanitários excepcionais frente à pandemia e relativos a medicamentos controlados são tema da Nota Orientativa n 14/2020, o uso da cloroquina, como terapia adjuvante no tratamento de formas graves da Covid-19 é abordado na Nota Orientativa $n^{\circ} 17 / 2020^{14}$.

A notificação de queixa técnica e evento adverso de medicamentos e produtos para a saúde no contexto da pandemia é tema abordado na Nota Orientativa ${ }^{\circ}$ $36 / 2020^{14}$.

Frente à importância de ações promotoras da saúde neste período, no qual o risco de doença é tema cotidiano, três notas orientativas foram publicadas ${ }^{14}$. 
A primeira, Nota Orientativa $n^{\circ} 15 / 2020$, trata da alimentação e atividade física. Considerando a importância da manutenção da saúde mental em época de pandemia, o tema é abordado na Nota Orientativa n 15/2020. E a violência e a atenção às pessoas em situação de violência no contexto da pandemia são tratadas na Nota Orientativa $n^{\circ} 30 / 2020^{14}$.

\section{Dados epidemiológicos}

A elaboração e divulgação de dados epidemiológicos é considerada uma das ações mais importantes no cotidiano da pandemia, pois consiste em importante ferramenta para a socialização de informações sensíveis à população, profissionais e autoridades da saúde. Os dados epidemiológicos contribuem para que a população tenha uma visão realística da pandemia na sua região de moradia, bem como da evolução no Estado.

Essas informações foram reunidas, sistematicamente, em Boletim Epidemiológico, sendo que o primeiro foi publicado dia 29 de fevereiro, quando havia oito casos de Covid-19 em investigação, todos com histórico de viagem ao exterior, sendo um residente fora do Estado do Paraná. A partir de março o Boletim Epidemiológico passou a ser publicado, diariamente, com dados do mundo, do Brasil e do Estado do Paraná, por Regional de Saúde e informações sobre o número de casos em investigação, casos recuperados e casos com evolução para o óbito. Os dados são estratificados por sexo e faixa etária, e os óbitos são também apresentados por município de residência.

\section{CONSIDERAÇÕES FINAIS}

A apresentação e contextualização do planejamento e ações desenvolvidas, no referido recorte temporal, no enfrentamento à pandemia no Paraná, traçam a linha de ação desenvolvida pela Secretaria Estadual de Saúde. Os temas pautados e discutidos permitiram o amadurecimento progressivo frente a demanda inédita, deixando um legado de experiência e aprendizado, aplicável não 
somente até o final desta pandemia, mas sobretudo para situações futuras de saúde pública.

A organização do COE e a determinação de seu núcleo central, com o apoio dos colaboradores e representantes institucionais, viabilizou a análise e aprovação de mais de 50 documentos, esses aplicáveis em diferentes esferas do sistema de saúde paranaense. Sua ação proativa e em tempo oportuno contribuiu para a organização e direcionamento dos serviços de saúde e da sociedade organizada no enfrentamento da pandemia por Covid-19.

Finalizada a pandemia, pesquisas poderão ser desenvolvidas contemplando a completude das ações realizadas, bem como de seu impacto para o sistema de saúde.

\section{REFERÊNCIAS}

1. Organização Pan-Americana da Saúde (OPAS). OMS afirma que COVID-19 é agora caracterizada como pandemia [Internet]. OPAS; 2020 [citado em 2020 Abr 20]. Disponível em: https://www.paho.org/bra/index.php?option=com_content\&view=artic le\&id=6120:omsafirma-que-covid-19-e-agora-caracterizada-como-pandemia\&ltemid=812.

2. Agência Nacional de Vigilância Sanitária (BR). Regulamento Sanitário Internacional RSI- 2005. Versão em português aprovada pelo Congresso Nacional por meio do Decreto Legislativo 395/2009 publicado no DOU de 10/07/09, pág.11.

3. Agência Nacional de Vigilância Sanitária (BR). Orientações e prioridades para ações de mitigação de riscos de descontinuidade de processos e atividades essenciais no contexto da pandemia de COVID-19. Versão 01 , de 6 de abril de 2020 [Internet]. Brasília; 2020 [citado em 2020 Abr 30]. Disponível em: http://portal.anvisa.gov.br/documents/219201/5777769/ Plano+de+Conting\%C3\%AAncia+GRC/ed86f520-92ee-4410-a2ff-e3d98f82a113.

4. Ministério da Saúde (BR). Plano de Contingência Nacional para Infecção Humana pelo novo Coronavírus COVID-19 Centro de Operações de Emergências em Saúde Pública | COE-COVID-19 [Internet]. Brasília; 2020. [citado em 2020 Mai 18]. Disponível em: https://www.saude.gov.br/images/pdf/2020/fevereiro/13/plano-contingencia-coronavirus-COVID19.pdf

5. Ministério da Saúde (BR). Portaria n 454, de 20 de março de 2020. Declara, em todo o território nacional, o estado de transmissão comunitária do coronavírus (Covid-19). Publicada no DOU 55, de 20 de março de 2020.

6. Paraná (BR). Plano de resposta a emergências em saúde pública do estado do Paraná [Internet]. Curitiba; 2020 [citado em 2020 Jun 30]. Disponível em: http://www.saude.pr.gov.br/sites/default/arquivos_restritos/files/documento/2020-04/ planoderespostaespfinal310718.pdf.

7. Polit DF, Beck CT. Fundamentos de pesquisa em enfermagem: avaliação de evidências para a prática da enfermagem. 9. ed. Porto Alegre: Artmed, 2011. 670 p.

8. Secretaria de Estado da Saúde do Paraná (PR). Resolução SESA Nº 0126/2020. Ativa o Comitê de Operações de Emergência em Saúde Pública (COE-COVID-19) [Internet]. Curitiba: SESA; 2020. [citado em 2020 Jun 30]. Disponível em:

http://www.saude.pr.gov.br/sites/default/arquivos_restritos/files/documento/2020-04/126_20.pdf.

9. Estado daSaúde do Paraná(PR). Decreton 4942/2020. Dispõesobremedidas restritivas regionalizadas para o enfrentamento da COVID-19. Publicado no Diário Oficial n. 10717 de 30 de junho de 2020 [Internet]. Curitiba; 2020 [citado em 2020 Jul 30]. Disponível em: https://www.legislacao.pr.gov.br/legislacao/listarAtosAno.do?action=exibirlmpressao\&codAto=235741. 
10. Secretaria de Estado da Saúde do Paraná (PR). Resolução SESA n 317/2020. Amplia a composição do COE [Internet]. Curitiba: SESA; 2020 [citado em 2020 Jun 30]. Disponível em: http://www.saude.pr.gov.br/sites/default/arquivos_restritos/ files/documento/2020-04/317_20.pdf.

11. Secretaria de Estado da Saúde do Paraná (PR). Resolução SESA nº 338/2020. Regulamenta o disposto nos arts. $1^{\circ}$ $2^{\circ}, 3^{\circ}, 10,13$ e 15 do Decreto Estadual $n^{\circ}$ 4.230, 16 de março de 2020, para implementar medidas de enfrentamento da emergência em saúde pública de importância nacional e internacional decorrente do Coronavírus - COVID-2019 [Internet]. Curitiba: SESA; 2020 [citado em 2020 Ago 6]. Disponível em: http://www.saude.pr.gov.br/sites/default/arquivos_restritos/ files/documento/2020-04/338_20.pdf.

12. Secretaria de Estado da Saúde do Paraná (PR). Decreto n 4230/2020. Dispõe sobre as medidas para enfrentamento da emergência de saúde pública de importância internacional decorrente do Coronavírus - COVID-19. Dispõe sobre as medidas para enfrentamento da emergência de saúde pública de importância internacional decorrente do Coronavírus - COVID-19 [Internet]. Curitiba: SESA; 2020 [citado em 2020 Ago 6]. Disponível em: https://www.legisweb.com.br/legislacao/?id=390948.

13. Secretaria de Estado da Saúde do Paraná (PR). Resolução SESA n 340/2020. Estabelece ações para contratação emergencial e institui recursos de custeio para oferta de leitos de Unidade de Terapia Intensiva e de Retaguarda Clínica para atendimento de usuários do Sistema Único de Saúde - SUS, com quadro clínico compatível com a infecção por Coronavírus - COVID-19, no Estado do Paraná [Internet]. Curitiba: SESA; 2020. [citado em 2020 Ago 6]. Disponível em: http://www.saude. pr.gov.br/sites/default/arquivos_restritos/files/documento/2020-04/340_20_consolidada.pdf.

14. Secretaria de Estado da Saúde do Paraná (PR). Notas Orientativas [Internet]. Curitiba: SESA; 2020. [citado em 2020 Ago 6]. Disponível em: http://www.saude.pr.gov.br/Pagina/Coronavirus-COVID-19.

15. Secretaria de Estado da Saúde do Paraná (PR). Resolução SESA n 544/2020. Dispõe sobre os procedimentos para prorrogação do prazo de validade das Licenças Sanitárias no Estado do Paraná em decorrência da pandemia de COVID-19, e dá outras providências [Internet]. Curitiba: SESA; 2020. [citado em 2020 Ago 6]. Disponível em: http://www.saude.pr.gov. br/sites/default/arquivos_restritos/files/documento/2020-04/544_20.pdf.

16. Secretaria de Estado da Saúde do Paraná (PR). Resolução SESA n 632/2020. Dispõe sobre medidas complementares de controle sanitário a serem adotadas para o enfrentamento da COVID-19 [Internet]. Curitiba: SESA; 2020. [citado em 2020 Ago 6]. Disponível em: http://www.saude.pr.gov.br/sites/default/arquivos_restritos/files/documento/2020-06/632_20.pdf.

17. Secretaria de Estado da Saúde do Paraná (PR). Resolução SESA n 856/2020. Edita ato normativo próprio estabelecendo normas e procedimentos para a regulamentação da retomada dos serviços essenciais e/ou não essenciais, em relação às medidas para a iniciativa privada acerca do enfrentamento da emergência de saúde pública de importância decorrente da COVID-19, conforme delegação disposta no Decreto Estadual n 4.545, de 27 de abril de 2020, art. $2^{\circ}$, que acrescentou $\mathrm{o}$ art. $2^{\circ} \mathrm{B}$ ao Decreto Estadual $n^{\circ}$ 4.317, de 21 de março de 2020 [Internet]. Curitiba: SESA; 2020. [citado em 2020 Ago 6]. Disponível em: https://www.documentador.pr.gov.br/documentador/pub.do?action=d\&uuid=@gtf-escriba-sesa@ d786c526-90c9-42b9-aca5-93b4d1ad8c39\&emPg=true.

18. Secretaria de Estado da Saúde do Paraná (PR). Resolução SESA nº 855/2020. Determina às indústrias de abate e processamento de carnes em todas as suas plantas frigoríficas a adoção de medidas de prevenção e controle da transmissão do SARS-CoV-2 no âmbito do Paraná [Internet]. Curitiba: SESA; 2020 [citado em 2020 Ago 6]. Disponível em: https://www. legisweb.com.br/legislacao/?id=397936.

19. Secretaria de Estado da Saúde do Paraná (PR). Nota Técnica nº5/2020. Disponibilização de Testes Rápidos para a detecção de anticorpos contra o coronavírus (SARS-CoV-2) e recomendações de grupos prioritários para a realização do teste [Internet]. Curitiba: SESA; 2020. [citado em 2020 Ago 6]. Disponível em: http://www.saude.pr.gov.br/sites/default/ arquivos_restritos/files/documento/2020-04/nota_tecnica_5_2020_dav_sesa.pdf.

20. Secretaria de Estado da Saúde do Paraná (PR). Nota Técnica nº 07/2020. Disponibilização de Testes Rápidos para a deteç̧ão de anticorpos contra o coronavírus (SARS-CoV-2) e recomendações de grupos prioritários para a realização da testagem [Internet]. Curitiba: SESA; 2020. [citado em 2020 Ago 6]. Disponível em: http://www.saude.pr.gov.br/sites/default/ arquivos_restritos/files/documento/2020-05/nota_tecnica_n7_2020_dav_sesa.pdf.

21. Secretaria de Estado da Saúde do Paraná (PR). Nota Técnica n. ${ }^{\circ}$ 07/2020 Complementação. Disponibilização de Testes Rápidos para a detecção de anticorpos contra o coronavírus (SARS-CoV-2) e recomendações de grupos prioritários para a realização da testagem [Internet]. Curitiba: SESA; 2020. [citado em 2020 Ago 6]. Disponível em: http://www.saude.pr.gov. br/sites/default/arquivos_restritos/files/documento/2020-06/NOTA_7_complementac\%CC\%A7a\%CC\%830_testes\%20 ra\%CC\%81 pidos.pdf.

22. Secretaria de Estado da Saúde do Paraná (PR). Nota Técnica Conjunta DAV/CHT SESA 01/2020. Disponibilização de Testes RT-PCR por meio de drive thru para profissionais e trabalhadores de saúde e de segurança pública [Internet]. Curitiba: SESA; 2020. [citado em 2020 Ago 6]. Disponível em: http://www.saude.pr.gov.br/sites/default/arquivos_restritos/ files/documento/2020-05/20200511161346946.pdf.

23. Secretaria de Estado da Saúde do Paraná (PR). Resolução SESA n 781/2020. Dispõe sobre a utilização de testes 
rápidos (ensaios imunocromatográficos) para a COVID19 em farmácias, e dá outras providências [Internet]. Curitiba: SESA; 2020 [citado em 2020 Ago 6]. Disponível em: http://www.saude.pr.gov.br/sites/default/arquivos_restritos/files/ documento/2020-06/781_20.pdf.

24. Secretaria de Estado da Saúde do Paraná (PR). Resolução SESA nº 482/2020. Regulamenta, em caráter excepcional e temporário, a operacionalização de prescrição médica por meio eletrônico, no contexto da pandemia de COVID-19 [Internet]. Curitiba: SESA; 2020. [citado em 2020 Ago 06]. Disponível em: http://www.saude.pr.gov.br/sites/default/arquivos_ restritos/files/documento/2020-04/482_20.pdf.

RECEBIDO: 30/07/2020

ACEITO: 07/10/2020 